\title{
Differentially expressed microRNAs in exosomes of patients with breast cancer revealed by next-generation sequencing
}

\author{
HEMING WU ${ }^{1-3 *}$, QIUMING WANG ${ }^{4 *}$, HUA ZHONG ${ }^{1-3}$, LIANG LI $^{4}$, \\ QUNJI ZHANG ${ }^{1-3}$, QINGYAN HUANG ${ }^{1-3}$ and ZHIKANG YU ${ }^{1-3}$ \\ ${ }^{1}$ Center for Precision Medicine, Meizhou People's Hospital (Huangtang Hospital), Meizhou Academy of Medical Sciences, \\ Meizhou Hospital Affiliated to Sun Yat-sen University; ${ }^{2}$ Guangdong Provincial Key Laboratory of Precision Medicine, \\ Clinical and Translational Research in Hakka Population, Meizhou People's Hospital (Huangtang Hospital), \\ Meizhou Academy of Medical Sciences, Meizhou Hospital Affiliated to Sun Yat-sen University; \\ ${ }^{3}$ Guangdong Provincial Engineering and Technology Research Center for Clinical Molecular Diagnostics \\ and Antibody Therapeutics; ${ }^{4}$ Center for Cancer Prevention and Treatment, \\ Meizhou People's Hospital (Huangtang Hospital), Meizhou Academy of Medical Sciences, \\ Meizhou Hospital Affiliated to Sun Yat-sen University, Meizhou, Guangdong 514031, P.R. China
}

Received May 10, 2019; Accepted October 10, 2019

DOI: $10.3892 /$ or.2019.7401

\begin{abstract}
MicroRNAs (miRNAs/miRs) in exosomes play crucial roles in the onset, progression and metastasis of cancer by regulating the stability of target mRNAs or by inhibiting translation. In the present study, differentially expressed miRNAs were identified in exosomes of 27 breast cancer patients and 3 healthy controls using RNA sequencing. The differentially expressed microRNAs were selected by bioinformatic analysis. Subjects were followed up for 2 years and exosomal miRNA profiles were compared between patients with and without recurrence of breast cancer. A total of 30 complementary DNA libraries were constructed and sequenced and 1,835 miRNAs were detected. There were no significant differences in the expression of miRNAs between the basal-like, human epidermal growth factor receptor- $2^{+}$, luminal A, luminal B and healthy control (HC) groups. A
\end{abstract}

Correspondence to: $\mathrm{Dr}$ Heming $\mathrm{Wu}$, Center for Precision Medicine, Meizhou People's Hospital (Huangtang Hospital), Meizhou Academy of Medical Sciences, Meizhou Hospital Affiliated to Sun Yat-sen University, 63 Huangtang Road, Meijiang, Meizhou, Guangdong 514031, P.R. China

E-mail: wuheming1986@126.com

*Contributed equally

Abbreviations: TNBC, triple-negative breast cancer; RT-qPCR, reverse transcription quantitative PCR; GO, Gene Ontology; KEGG, Kyoto Encyclopedia of Genes and Genomes; FC, fold change; ANOVA, one-way analysis of variance; ROC, receiver operating characteristic

Key words: microRNAs, exosomes, breast cancer, triple-negative breast cancer, next-generation sequencing total of 54 differentially expressed miRNAs were identified in triple-negative breast cancer (TNBC) patients vs. HCs, including 20 upregulated and 34 downregulated miRNAs. The results of the reverse transcription-quantitative PCR were consistent with this. Receiver operating characteristic curve analyses indicated that miR-150-5p [area under the curve $(\mathrm{AUC})=0.705$, upregulated $], \mathrm{miR}-576-3 \mathrm{p}(\mathrm{AUC}=0.691$, upregulated), $\mathrm{miR}-4665-5 \mathrm{p}$ (AUC $=0.681$, upregulated) were able to distinguish breast cancer patients with recurrence from those without recurrence. In conclusion, the present results indicated differences in miRNA expression profiles between patients with TNBC and healthy controls. Certain exosomal miRNAs were indicated to have promising predictive value as biomarkers for distinguishing breast cancer with recurrence from non-recurrence, which may be utilized for preventive strategies.

\section{Introduction}

Breast cancer is one of the leading causes of cancer-associated mortality among females worldwide, which affects about one in eight females in developed countries $(1,2)$. Increased incidence rates and mortality rates have been reported in most developed and developing countries, respectively (3). In China, the health burden of cancer is increasing. Every year, more than 1.6 million individuals are diagnosed with breast cancer and 1.2 million patients succumb to breast cancer. In numerous countries, including China, breast cancer is now the most common type of cancer diagnosed in females (4).

Numerous studies have confirmed that different molecular subtypes of breast cancer differ in their clinicopathological characteristics, differ in regards to the benefit from various chemotherapy regimens and differ in regards to patient prognosis (5-7). Breast cancer has been classified into distinct subtypes, including luminal, human epidermal growth factor receptor-2 (Her-2)-enriched and basal-like (8,9). In 2003, 
Sorlie et al $(10,11)$ divided the luminal type into two subtypes of luminal A and luminal B.

Luminal subtype A is defined as estrogen receptor (ER)or progesterone receptor (PR)-positive, Her-2-negative and Ki67 low expression as determined by immunohistochemistry. This type is the most common subtype of breast cancer and is associated with the best prognosis (12). Luminal subtype $B$ is a subtype of breast cancer with ER- or PR-positive status, either positive or negative regarding Her-2 and high expression of Ki67. Luminal subtype B is most commonly identified in elderly patients with breast cancer, where ductal carcinoma is common (13). Her-2-positive breast cancer is Her-2-positive upon immunohistochemistry (3+) or fluorescence in situ hybridization, negative for ER and PR, and has high expression of Ki67. The molecular characteristics of Her-2-positive breast cancer are obvious amplification of the erb-b2 receptor tyrosine kinase 2 (ERBB2) gene, and upregulation of associated genes, including growth factor receptor bound protein 7 (GRB7) and TRAP100 $(14,15)$. Basal-like is a molecular subtype that has been studied more frequently. Its characteristics are lack of ER and Her-2 expression and high expression of the biomarkers of the basal epithelium, which is derived from the outer layer of the ductal epithelium $(16,17)$. Basal-like breast cancer and triple-negative breast cancer (TNBC) are basically the same subtypes. Basal-like is a subtype of breast cancer identified according to the gene expression profile, while TNBC is identified by immunohistochemistry and exhibits certain differences in its characteristics $(18,19)$.

TNBC refers to breast cancer with negative ER, PR and proto-oncogene Her-2 upon immunohistochemical examination of cancer tissues. This type of breast cancer accounts for $12-17 \%$ of all pathological types of breast cancer, with a distinct biological behavior and clinicopathological characteristics, and a worse prognosis than other types (20). According to research, the local recurrence of TNBC is not significantly different from that of non-TNBC, but the incidence of distant metastasis is higher. The incidence rates of lung metastasis and liver metastasis were reported to be high, but there was no difference regarding bone and brain metastasis (21-23). It is important to identify biomarkers to predict the sensitivity to chemotherapy and prognosis of TNBC patients.

Exosomes are described as vesicles of $40-100 \mathrm{~nm}$ in diameter, which are secreted by certain cells (24). Exosomes may be secreted by tumor cells during tumor progression and metastasis. They have been reported to have vital roles in the progression and metastasis of the tumor by communication via molecules including lipids, proteins, mRNAs and microRNAs (miRNAs/miRs) (25-27). Exosomes may hold promise as potential biomarkers, and separation and detection of circulating tumor-associated exosomes may be used for the diagnosis of cancer patients (28). Blocking of exocrine secretions may inhibit the occurrence of tumors (29).

miRNAs are a type of non-coding, conserved and single-stranded RNAs consisting of 18-24 nucleotides. Mature single-stranded miRNA fragments are integrated with the argonaut protein (Ago2) and target mRNA to form the RNA-mediated silencing complex to regulate post-transcriptional gene expression by either inhibiting the translation process or promoting the degradation process of the target
mRNA $(30,31)$. miRNAs regulate the stability of target mRNAs or inhibit translation to control cell proliferation, differentiation, invasion, migration and apoptosis, and have a key role in tumorigenesis and progression (32). In the present study, RNA sequencing was applied to detect tumor-specific miRNAs in exosomes that may be associated with the onset and progression of breast cancer.

\section{Materials and methods}

Subjects. All of the subjects included in the present study presented themselves to the Cancer Center of Meizhou People's Hospital (Meizhou, China) for medical examination between October 2015 and January 2016. The patients were aged between 30 and 69 years and their mean age was $49.83 \pm 9.763$ years. The study was performed on the basis of the Declaration of Helsinki, and was supported by the Ethics Committee of Meizhou People's Hospital.

Sample collection and total RNA extraction. Two peripheral blood samples were taken from each subject at the same time; $4 \mathrm{ml}$ was used for detection of tumor markers, while $5.0 \mathrm{ml}$ of peripheral venous blood was collected in tubes with EDTA anti-coagulant for detection of miRNAs from patients with breast cancer and healthy controls. Plasma was separated and transferred to 1.5-ml RNA centrifuge tubes (RNase-free) and then packaged and stored at $-80^{\circ} \mathrm{C}$ for exosome isolation and RNA extraction. Prior to sampling, the subjects did not receive any pre-operative radiotherapy and/or chemotherapy and were diagnosed with breast cancer by pathology.

Exosome isolation was performed using Exosome Isolation Reagent (RiboBio Co., Ltd.) according to the manufacturer's protocol. Total RNA was extracted from the exosomes using TRIzol reagent (Invitrogen, Thermo Fisher Scientific, Inc.) according to the manufacturer's protocol. The quantity and purity of total RNA were evaluated with a Nanodrop 2000 (Thermo Fisher Scientific, Inc.), and the RNA Nano 6000 Assay Kit of the Agilent Bioanalyzer 2100 system (Agilent Technologies) was used to analyze RNA integrity.

Library construction and high throughput sequencing. Library construction of small RNA was performed with the Illumina TruSeq small RNA sample pre Kit (Illumina, Inc.), with which joints were directly added to the specific structure of the $3^{\prime}$ and 5'end of the small RNA (5'end phosphate group, 3'end hydroxyl group). Subsequently, the RNA was reverse-transcribed to complementary (c)DNA. Following PCR amplification, the target DNA fragments were separated by PAGE, and the cDNA libraries were recovered and purified. PAGE was used to separate the target DNA fragments after PCR and recover and purify the cDNA library.

After the libraries were constructed, they were preliminarily quantified by Qubit2.0 and the libraries were diluted to $1 \mathrm{ng} / \mu \mathrm{l}$. An Agilent 2100 (Agilent Technologies, Inc.) was used to detect the library size. The insert size was in line with the expectations, and accurate quantification of the effective concentration of the libraries (libraries with an effective concentration of $>2 \mathrm{nM}$ ) was performed using quantitative (q)PCR in order to ensure the quality of the libraries. After the qualification of the libraries, the different libraries were 
sequenced on the Illumina HiSeq 2500 platform (Illumina, Inc.) according to the protocols of RiboBio Co., Ltd.

Identification of differentially expressed miRNAs. The adapter sequences and low-quality sequences were removed prior to data analysis. The remaining reads were referred to as 'clean reads' and acquired for the transcriptome assembly and quantification. Next, the index of the reference genome was built using Bowtie v2.0.6 (http://bowtie-bio. sourceforge.net) and paired-end clean reads were mapped to the reference genome using TopHat v2.0.9 (http://ccb. jhu.edu/software/tophat/index.shtml). The transcriptome assemblies were then generated using Cufflinks v2.1.1 (https://cole-trapnell-lab.github.io/cufflinks) with the default parameters. The miRNA sequencing reads of each sample were normalized to fragments per kilobase of transcript per million mapped reads values using Cuffdiff v2.1.1 (http://cole-trapnell-lab.github.io/cufflinks). The P-value and fold change were calculated for each gene. $\mathrm{P}<0.05$ and $\mid \log _{2}$ (foldchange) $\mid>1$ were set as the threshold for significantly differential expression.

Gene Ontology (GO) and Kyoto Encyclopedia of Genes and Genomes (KEGG) enrichment analysis. GO analysis was performed to elucidate the potential biological process terms of the differentially expressed genes in GO annotations. Furthermore, pathway analysis was utilized to screen out the significant pathways of the differentially expressed genes according to the KEGG database. Fisher's exact test was applied to evaluate whether the GO terms or the KEGG pathways were enriched by the differentially expressed genes, and $\mathrm{P}<0.05$ was set as the threshold of significant difference.

Reverse transcription (RT)-qPCR. Differentially expressed miRNAs were randomly selected and their expression levels were detected by RT-qPCR. To validate the sequencing results regarding differentially expressed miRNAs, six upregulated miRNAs [Homo sapiens (hsa)-miR-148a-5p, hsa-miR-200a-5p, hsa-miR-210a-3p, hsa-miR-378a-3p, hsa-miR-483-5p and hsa-miR-7110-5p] and two downregulated miRNAs (hsa-miR-92b-3p and hsa-miR-150-5p) were selected. The qPCR forward primer sequences were as follows: 5'-AAGTTCTGAGACACTCC-3', 5'-CATCTTACC GGACAGT-3', 5'-TGTGACAGCGGCTAA-3', 5'-CTGGAC TTGGAGTCAGAAG-3', 5'-AGACGGGAGGAAAGAA-3', 5'-TGTGGGGAGAGAGAG-3', 5'-TATTGCACTCGTCCC G-3' and 5'-CAACCCTTGTACCAGTG-3', respectively. Total RNA from exosomes was extracted using an RNeasy Kit (TianGene Co., Ltd.) and reverse-transcribed to cDNA using the following conditions: $42^{\circ} \mathrm{C}$ for $1 \mathrm{~h}, 70^{\circ} \mathrm{C}$ for $10 \mathrm{~min}$ and a hold at $4^{\circ} \mathrm{C}$. The cDNA was amplified using miRNA assay primers and the SYBR Green Mix (RiboBio Co., Ltd.) on the Roche Lightcycler 480 (Roche Molecular Systems, Inc.). In addition, a common reverse primer was used in the present study, with the sequence: 5'-CAGTGCGTGTCGTGGAGT-3'. Each reaction was performed under the following conditions: initialization for $10 \mathrm{~min}$ at $95^{\circ} \mathrm{C}$, followed by 40 cycles of amplification, with $2 \mathrm{sec}$ at $95^{\circ} \mathrm{C}$ for denaturation, $20 \mathrm{sec}$ at $60^{\circ} \mathrm{C}$ for annealing, and $10 \mathrm{sec}$ at $70^{\circ} \mathrm{C}$ for elongation. The expression of exosomal miRNAs was quantified using the $2^{-\Delta \Delta C q}$ (33) method and all miRNA expression values were normalized against the U6 internal control.

Comparison of exosomal miRNA profiles between patients with and without recurrence of breast cancer. The 27 subjects were followed up for 2 years. The differential expression of miRNAs in patients with and without recurrence of breast cancer was analyzed. Blood samples were collected from all of these patients at the time-point of diagnosis. Based on the follow-up results, the differential expression of miRNAs at the initial diagnosis was analyzed, aiming to identify miRNAs at the time-point of initial diagnosis that are able to predict the presence of metastasis.

Statistical analysis. SPSS statistical software version 19.0 (IBM Corp.) was used for data analysis. Values are expressed as the mean \pm standard deviation. Chi-square was used to compare the frequencies. Multigroup comparisons of the means were carried out by one-way analysis of variance (ANOVA) test with post hoc contrasts test were used to determine the statistical significance of differences between groups. $\mathrm{P}<0.05$ was considered to indicate statistical significance. Receiver operating characteristic (ROC) curve analysis was performed and the area under the ROC curve (AUC) was calculated to determine the diagnostic value of certain differentially expressed miRNAs randomly selected for differentiating between breast cancer patients and healthy controls.

\section{Results}

Clinical characteristics. A total of 27 breast cancer patients and 3 healthy controls participated in the present study. The clinical characteristics of the 30 subjects of this study, including age, clinical stage, immunohistochemistry, tumor markers and blood lipid levels, are presented in Table SI.

Statistics of the sequencing data. A total of 395,775,015 raw reads were obtained, with an average of 13,192,500 raw reads for each sample. After data filtering and normalization, 372,788,760 clean reads were obtained, with an average 12,426,292 clean reads for each sample. The Q30 mean value was $94.19 \%$, with a range of $91.13-97.83 \%$ for each sample. This indicated that the quality of these 30 libraries was suitable for the subsequent analysis. Detailed information on the quality of the sequencing data is presented in Table SII.

Differentially expressed miRNAs in exosomes. miRNAs were analyzed with strict quality control of the data, and a total of 1,835 miRNAs were identified. In order to systematically study the level of miRNA expression associated with breast cancer, 27 patients with breast cancer and 3 healthy controls were analyzed in the present study. Hierarchical clustering of the miRNAs in breast cancer patients and healthy controls is provided in Fig. 1 .

Volcano plots were used to analyze the overall distribution of differentially expressed miRNAs. The fold change and corrected significance level were used to screen differentially expressed miRNAs. No significant differences in miRNAs were identified among the basal-like, HER- $2^{+}$, luminal A, luminal $\mathrm{B}$ and $\mathrm{HC}$ groups. Compared to the miRNA expression 


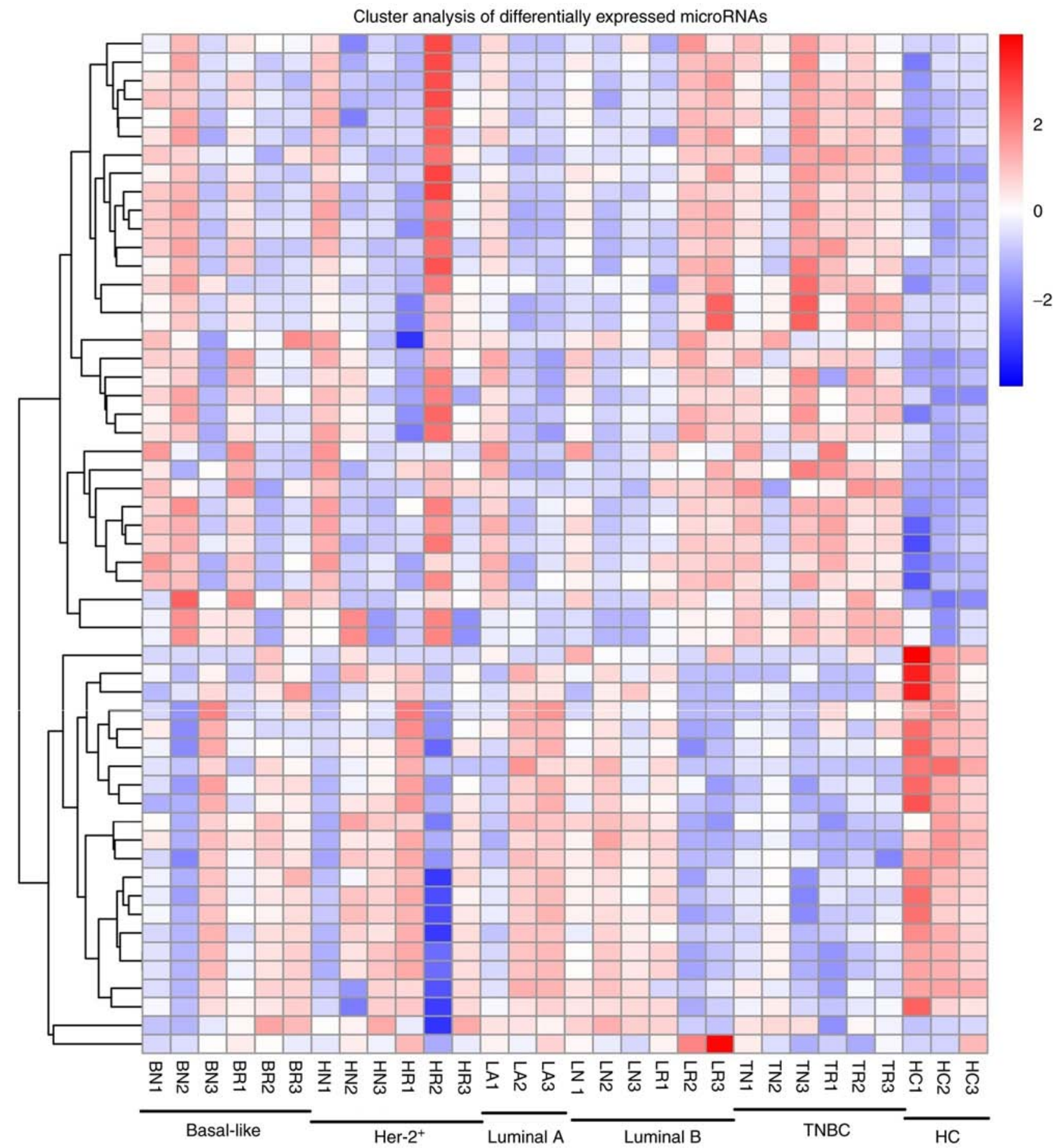

Figure 1. Heat map of the microRNAs in breast cancer patients and healthy controls. miRNA expression is hierarchically clustered on the y-axis, and plasma samples from breast cancer patients (basal-like, $n=6$; HER-2 $2^{+}, n=6$; luminal $A, n=3$; luminal $B, n=6 ;$ TNBC, $n=6$ ) and healthy controls $(n=3$ ) are hierarchically clustered on the $\mathrm{x}$-axis. The red and the blue shades indicate an increase and a decrease in expression level, respectively. TNBC, triple negative breast cancer; $\mathrm{HC}$, healthy control.

profiles of the healthy controls, a total of 54 differentially expressed miRNAs were discriminated in TNBC patients, including 20 upregulated miRNAs and 34 downregulated miRNAs $(\mathrm{P}<0.05)$. A volcano plot is displayed in Fig. 2 and a list of miRNAs identified to be differentially expressed between TNBC patients and HCs is provided in Table SIII. The blue and red in the figure indicate that reduced and increased expression, respectively. The frequency of base usage among miRNA sequences is indicated in Fig. 3.
GO and KEGG pathway analyses. To study the functions and associated pathways of the miRNAs identified in the present study, GO and pathway analyses were performed. The hierarchical classification of genes in the categories cellular component, molecular function of biological process revealed gene regulatory networks.

TNBC-associated miRNAs were selected and the biological process pathways enriched by these miRNAs were studied. Analysis indicated statistically significant accumulation of 


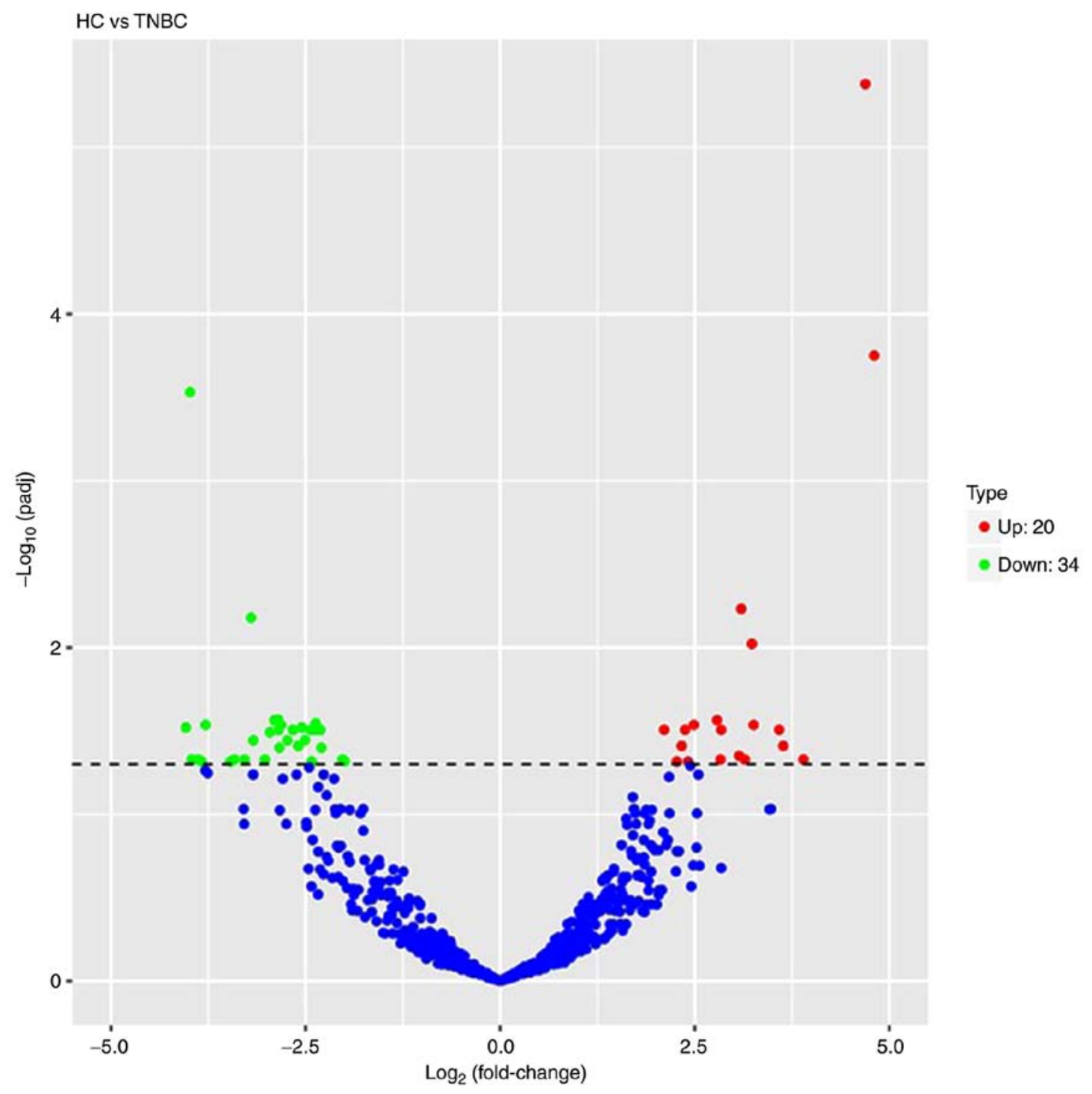

Figure 2. Volcano plot of differential microRNA expression. $x$-axis, $\log _{2}$ (fold change); y-axis, $-1 \operatorname{xlog}_{10}$ (padj) for each probes. The scattered dots represent the individual microRNAs, the blue dots represent the microRNAs with no significant difference, the red dots represent the significantly upregulated differential microRNAs, and the green dots represent the significantly downregulated differential microRNAs. TNBC, triple-negative breast cancer; HC, healthy control
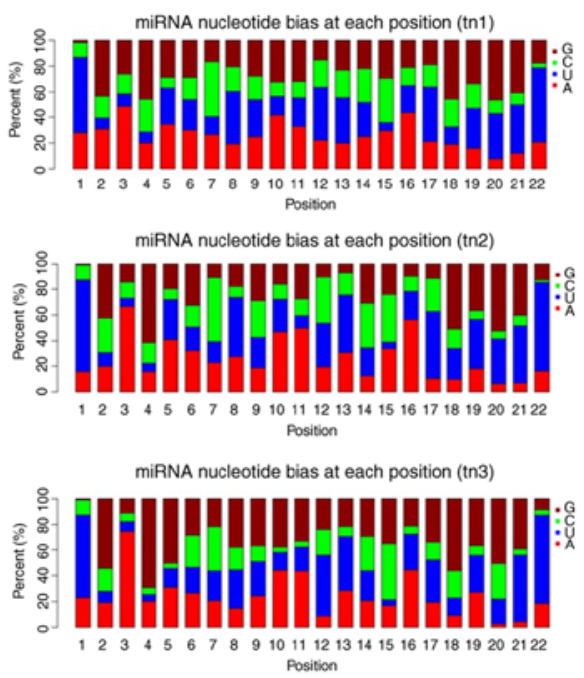

TNBC group

$$
\begin{array}{ll}
11 & 1213 \\
\text { Position }
\end{array}
$$
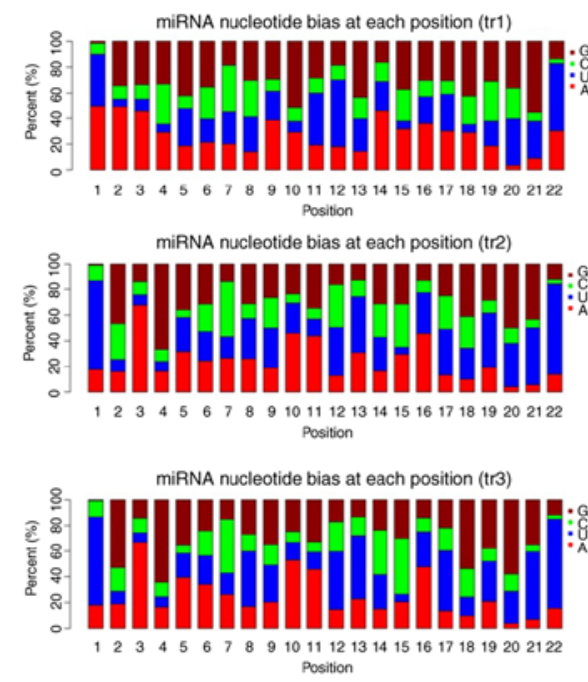

Position

Figure 3. Frequency of base usage among microRNA sequences. The abscissa of which is the base position of the microRNA, and the ordinate of which is the percentage of base A/U/C/G present in the microRNA. Red indicates A; blue, U; green, C; brown, G. TNBC, triple-negative breast cancer; HC, healthy control. 


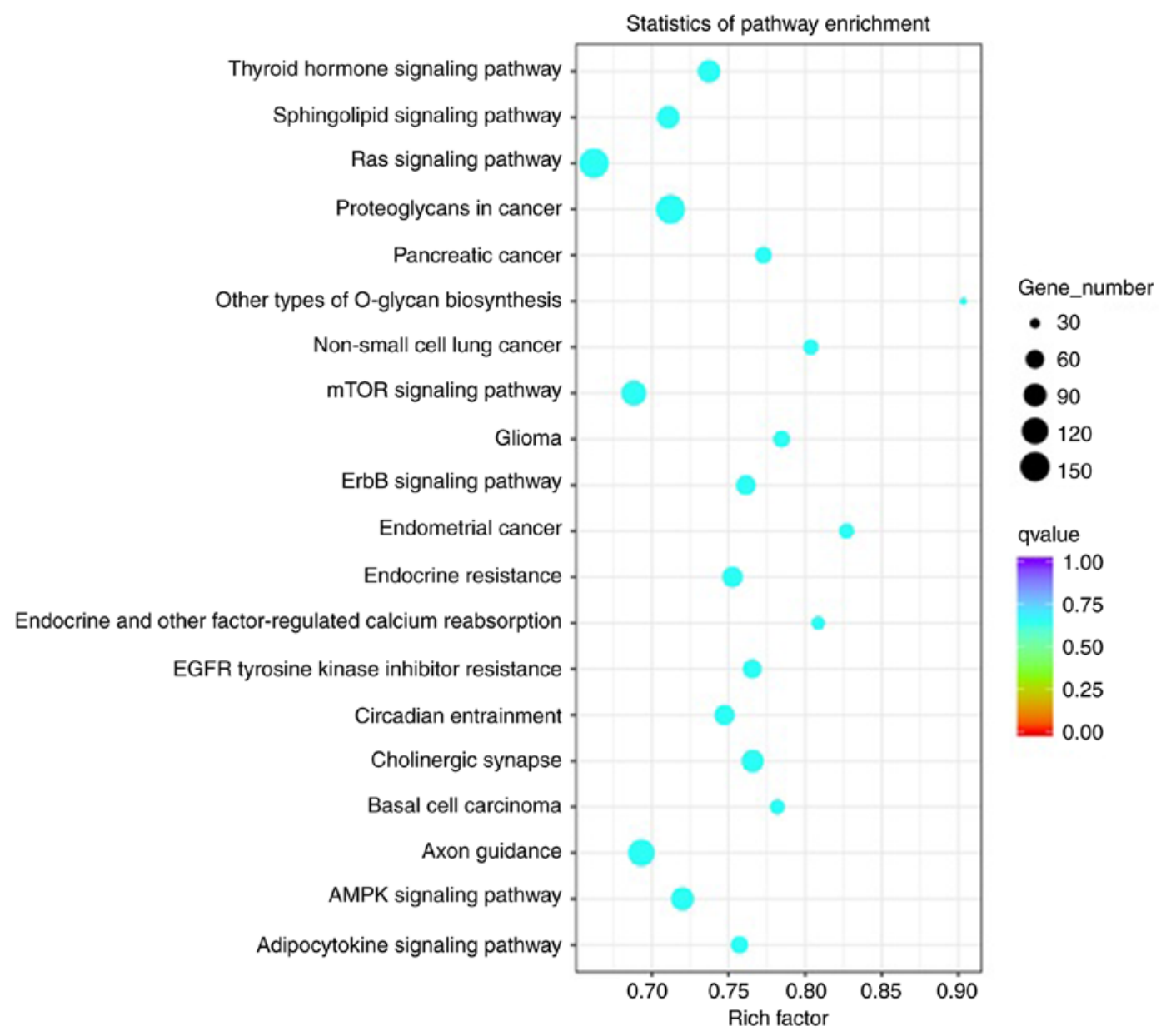

Figure 4. Pathway analysis of differentially expressed microRNAs. Kyoto Encyclopedia of Genes and Genomes analysis of target genes of differentially expressed microRNAs. The lower the P-value, the more significant the pathway.

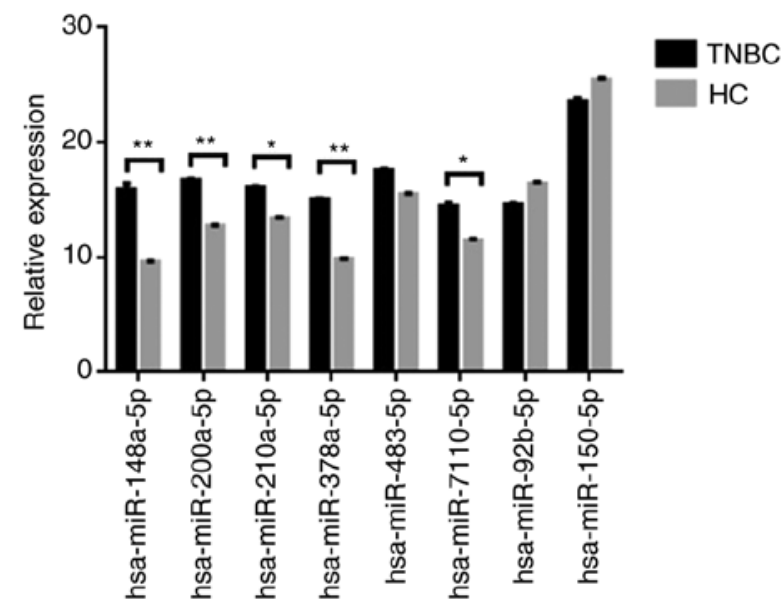

Figure 5. Validation of RNA-seq results by RT-qPCR. Eight differentially expressed microRNAs were selected and examined by RT-qPCR. The fold change from RT-qPCR was determined using the $2^{-\Delta \Delta C q}$ method and all microRNA expression values were normalized against the U6 internal control. Data from RT-qPCR are shown as mean \pm standard deviation (SD) of one representative experiment. The data are expressed as the mean $\pm \mathrm{SD}$ $(\mathrm{n}=20)$. TNBC, triple-negative breast cancer; HC, healthy control.

pathways linked to proliferation, signal transduction and migration, and the associated functions are presented in Fig. 4. These pathways included the 'Ras signaling pathway', 'mTOR signaling pathway', 'Axon guidance', 'proteoglycans in cancer', 'sphingolipid signaling pathway', 'adenosine monophosphate kinase (AMPK) signaling pathway' and 'thyroid hormone signaling pathway'.

$R T-q P C R$ validation of miRNA expression. To validate the sequencing data for certain miRNAs, the expression levels of six upregulated miRNAs (hsa-miR-148a-5p, hsa-miR-200a-5p, hsa-miR-210a-3p, hsa-miR-378a-3p, hsa-miR-483-5p and hsa-miR-7110-5p) and two downregulated microRNAs (hsa-miR-92b-3p and hsa-miR-150-5p) in the exosome of TNBC patients $(n=20)$ and healthy controls $(n=20)$ were verified by RT-qPCR. Details concerning the patient collective used for verification of the data are provided in Table SI. U6 small nuclear RNA was used as the internal control. As presented in Fig. 5, there were significant differences in the expression of eight miRNAs between TNBC patients and healthy controls. The results of RT-qPCR were consistent with those of the RNA sequencing.

Comparison of exosomal miRNA profiles between patients with and without recurrence of breast cancer and ROC analysis. Certain miRNAs were differentially expressed in exosomes of patients with vs. without recurrence. Hierarchical clustering of differentially expressed miRNAs between patients with and without recurrence in breast cancer is provided in Fig. 6. 


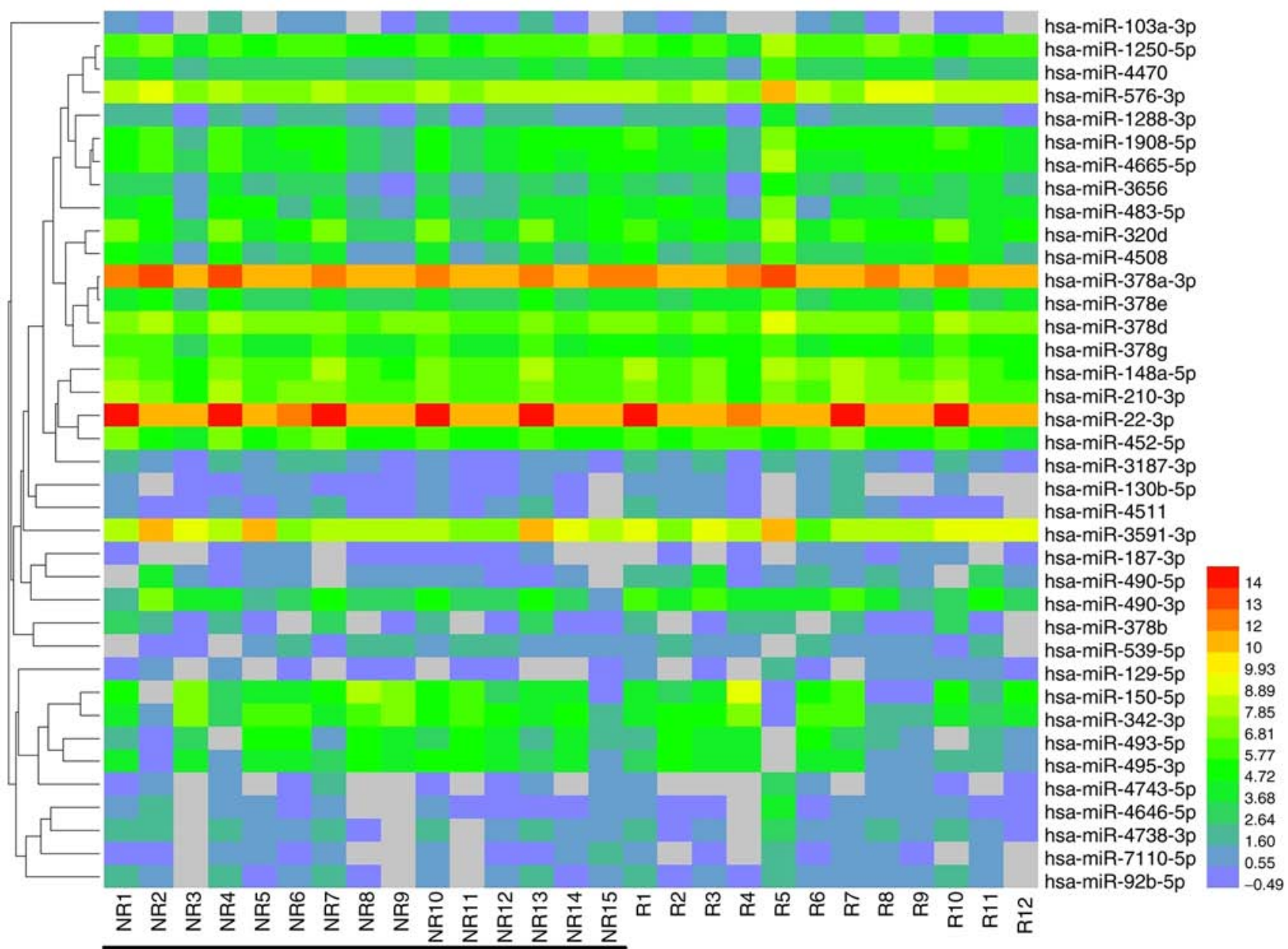

Non-recurrence

Recurrence

Figure 6. Hierarchical clustering of microRNAs between patients with and without recurrence in breast cancer. miRNA expression is hierarchically clustered on the $y$-axis, and plasma samples from breast cancer patients with and without recurrence are hierarchically clustered on the $\mathrm{x}$-axis. The red and the green shades indicate an increase and a decrease in expression level, respectively.
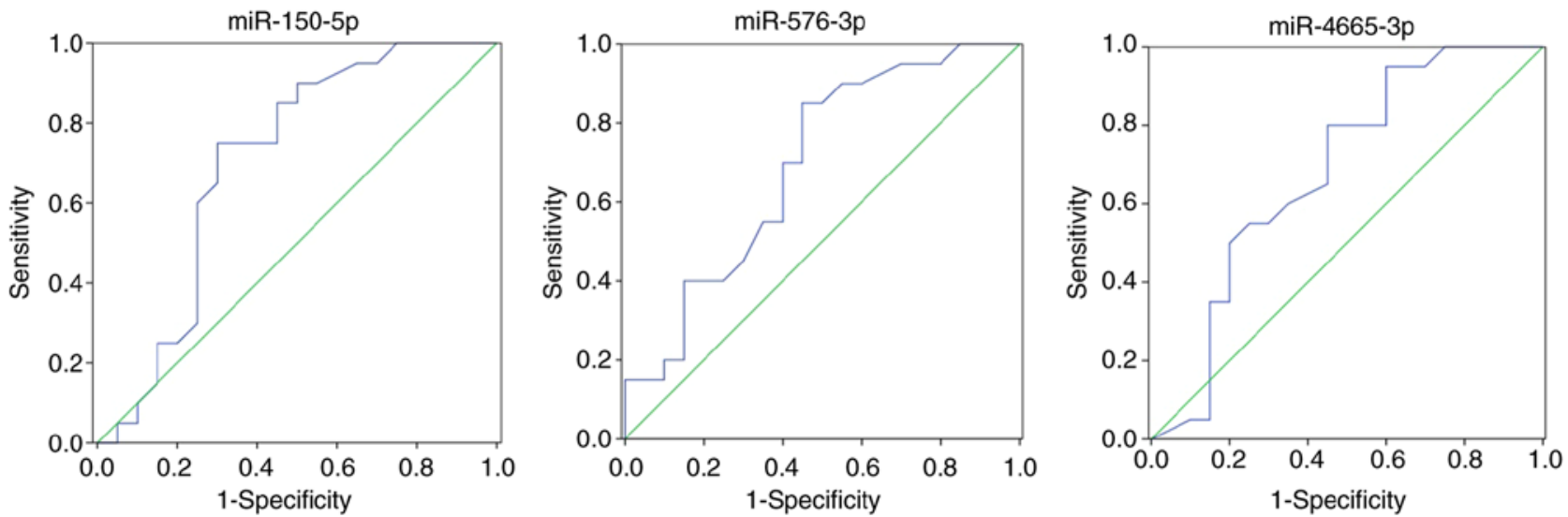

Figure 7. Three differentially expressed microRNAs in exosomes were randomly selected for differentiating between patients with and without recurrence in breast cancer to evaluate the diagnostic value by ROC analysis. Receiver operating characteristic (ROC) curves were generated to assess the diagnostic accuracy of these microRNAs, and the sensitivity and specificity of the optimum cut-off point were defined as those values that maximized the area under the ROC curve (AUC). MicroRNAs with a $\mathrm{P}<0.05$ and an AUC $>0.6$ were selected as successful distinguishing markers for patients with and without recurrence in breast cancer.

hsa-miR-150-5p, hsa-miR-576-3p and hsa-miR-4665-5p were considered as potential biomarkers in the patients with recurrence.
Certain differentially expressed miRNAs in the exosome were randomly selected for evaluating their ability to distinguish patients with recurrence of breast cancer from those 
without recurrence, and their diagnostic value was determined by ROC curve analysis. miRNAs with a $\mathrm{P}<0.05$ and an AUC $>0.6$ were selected as markers with the ability to distinguishing between patients with and without recurrence in breast cancer. More specifically, the ROC curves yielded the following AUCs: miR-150-5p (AUC=0.705, upregulated), miR-576-3p (AUC=0.691, upregulated), miR-4665-5p (AUC $=0.681$, upregulated). These miRNAs were identified to be able to discriminate breast cancer patients with recurrence from without recurrence. The ROC curves are provided in Fig. 7.

\section{Discussion}

The development of human cancers is a multi-step process in which normal cells acquire traits that eventually lead them to become cancer cells. The interaction between tumor cells and the surrounding microenvironment is key in overcoming this obstacle and promoting tumor progression and metastasis. Exosomes are membrane-derived vesicles that are regarded as important mediators for intercellular communication in recent years. They carry lipids, proteins, mRNAs and miRNAs and may be transferred to recipient cells by fusion of exosomes and target cell membranes. In the context of cancer cells, this process requires the transfer of pro-cancer cell content to surrounding cells within the tumor microenvironment or into the circulation to function at a distance to allow the cancer to progress. In this process, exosomal small RNAs are transferred to recipient cells, where they regulate the expression of target genes.

miRNAs are endogenous, non-coding RNAs that regulate gene expression mainly at the post-transcriptional level, and their expression depends on the physiological and pathological state of the body. To date, characteristic miRNA expression patterns have been identified in malignant tumors, which are different from those in normal tissues. Detection of these miRNAs may be an important means of early diagnosis, targeted therapy and prognostication of patients with malignant tumors. However, miRNAs are intracellular molecules that reside inside the cells, and it is difficult to assess the expression levels of miRNAs from the cells in deep tissues. miRNAs in exosomes are protected from exposure to nucleases and shear stress, and these characteristics make exosomal miRNAs potential markers for disease detection. It is well known that exosome-encapsulated miRNAs serve as ideal biomarkers for disease detection at an early stage.

In breast cancer patients, certain miRNAs may be abnormally expressed, thereby promoting the proliferation, survival or metastasis of tumor cells. For instance, miR-2l, miR-10b, and miR-155 are frequently overexpressed in breast cancer cells (34), and miR-30b, miR-126, miR-17P, miR-335 and miR-205 are frequently downregulated in breast cancer cells $(35,36)$. miRNAs that are abnormally expressed in breast cancer have multiple roles, but they may be divided into cancer-promoting or tumor-suppressor miRNAs. They exert their cancer-promoting or tumor-suppressing effects by affecting the corresponding signaling pathways and transcription factors. For instance, miR-21 exerts its promoting effect on the anti-apoptotic protein Bcl-2, as well as programmed cell death 4 and p53 (37), and its concentration in serum and breast cancer tissues is positively associated with metastasis and the poor prognosis of breast cancer patients. By contrast, miR-155, let-7e, miR-10a, miR-144 and miR-193 exert anti-apoptotic effects by acting on caspase-3 (38). Overexpression of miR-155 may also activate the JAK/STAT3 pathway and promote tumorigenesis and development. miR-10b promotes downregulation of E-cadherin expression (39). Others, including miR-27a, miR-96 and miR-182, may have a cancer-promoting role by inhibiting forkhead box O1 (FOXO1) (40).

Previous studies on the association between miRNAs and breast cancer were summarized and compared with the present results. By targeting GLUT1, miR-22 may inhibit the proliferation and invasion of breast cancer cells, and the expression of miR-22 and GLUT1 in breast cancer tissue samples exhibits a negative correlation (41). NF-E2-related factor 2 (Nrf2) is an important transcription factor that activates the expression of cellular detoxifying enzymes. miR-200a regulates the Keap1/Nrf2 pathway inmammary epithelium and re-activates the Nrf2-dependent anti-oxidant pathway in breast cancer $(42,43)$. Estradiol-G-protein-coupled estrogen receptor 1 induces the level of HOTAIR through the suppression of miR-148a. miR-148a levels were reported to be negatively correlated with HOTAIR levels in breast cancer patients (44-46). Studies have reported an association between miR-210 and neo-adjuvant chemotherapy in HER2-positive breast cancer $(47,48)$. Furthermore, the expression level of miR-378 was found to be highly associated with breast cancer. Overexpression of miR-378 in formalin-fixed and paraffin-embedded tissues of breast cancer patients may serve as an ideal diagnostic biomarker for breast cancer (49). miR-378a-3p was reported to modulate the sensitivity of breast cancer MCF-7 cells to tamoxifen through targeting Golgi transport 1A (50). Various studies suggest that treatment with miR-296 is effective in ER-negative breast cancers. A combination of let-7 with miR-296 may be an efficient molecular medicine for any type of breast cancer (51). The miR-320a/metadherin pathway is a putative therapeutic target in breast cancer and dysregulation of miR320a may be involved in invasive breast cancer progression $(52,53)$. Another study suggested that modulating the levels of miR-34a, a tumor-suppressor miRNA, may be a novel and useful approach for breast cancer therapy (54). The present study also indicated differential expression of the above mentioned miRNAs in breast cancer.

Among the miRNAs identified to be differentially expressed in the present study, certain miRNAs have been previously reported in breast cancer. Chabre et al (55) indicated that circulating miR-483-5p is a promising non-invasive biomarker for the clinical outcome of adrenocortical carcinoma. miR-486-5p was also reported to be downregulated in patients with lymph node metastases (56), lung adenocarcinoma (57) and breast cancer (58). miR-490-3p is downregulated in triple-negative breast cancer (TNBC) and has a suppressive role in cancer cell proliferation, invasion and tumorigenesis (59), and the present results were consistent with this finding. Lower expression levels of miR-378a-3p were reported to be associated with poor prognosis for tamoxifen-treated patients with breast cancer (50). Decreased expression of miR-122-5p was reported in patients with all stages and grades of breast cancer (60). Several studies found that miR-210 expression is associated with breast cancer patient survival (61). miR-320a was found to inhibit the proliferation, invasion (62) and metastasis (53) 
of breast cancer. miR-34a-5p was suggested as a potential biomarker of chemotherapy-associated cardiac dysfunction in breast cancer patients (63). Circulating miRNAs are potential biomarkers for distinguishing inflammatory breast cancer (IBC) from non-IBC, and may also be a candidate for early detection of breast cancer (64). High levels of miR-374b-5p were found to be associated with a favorable outcome for TNBC (65). The expression levels of miR-155-5p were reported to be upregulated in TNBC (66-68). The present results were consistent with these studies. The clinical outcome for patients with breast cancer presenting with positive miR-361-5p expression was significantly better than that of patients with negative miR-361-5p expression (69). Another study found that miR-361-5p is downregulated in TNBC tissues and that overexpression of miR-361-5p inhibits the proliferation, migration and invasion of TNBC cells (70). A further study indicated that miR-429 is upregulated in TNBC (71). miR-92a-3p may be part of target pathways for selective intervention to suppress hormone-regulated metastasis in breast cancer (72).

In the present study, TNBC-specific miRNAs were identified and subjected to GO functional enrichment analysis in the category biological process and KEGG pathway analysis, revealing that these miRNAs were significantly accumulated in pathways associated with proliferation, signal transduction and migration. Certain pathways were found to be associated with cancer, mainly including the 'Ras signaling pathway' $(73,74)$, 'mTOR signaling pathway' $(75,76)$, 'axon guidance' (77), 'proteoglycans in cancer' (78,79), 'sphingolipid signaling pathway' (80), 'AMPK signaling pathway' $(81,82)$, 'thyroid hormone signaling pathway' (83), 'ErbB signaling pathway' (84) and 'epidermal growth factor receptor tyrosine kinase inhibitor resistance' $(85,86)$.

ROC curve analysis was performed to assess the sensitivity and specificity of miRNAs as diagnostic biomarkers. The exosome levels of hsa-miR-150-5p, hsa-miR-576-3p and hsa-miR-4665-5p were higher in breast cancer with recurrence compared to those in patients without recurrence. Therefore, the results indicated that these exosomal miRNAs may serve as novel biomarkers with a predictive value for breast cancer recurrence. It is important to explore the association between miRNAs and the diagnosis, treatment and prognosis of breast cancer. However, current understanding of the regulatory mechanisms of miRNAs is still superficial and their interaction with other regulatory mechanisms requires further analysis. The present study may provide insight into the important roles of certain miRNAs in the occurrence and development of breast cancer.

As a diagnostic marker, exosomes are stable, capturable, bioactive and real-time. However, there are currently limitations regarding the application of exosomes as clinical markers, including specificity, source clarity, enrichment efficiency, detection methods and convenience of extraction. In addition to miRNAs, exosomal mRNA and protein will become a novel research 'hotspot'. Regarding precise applications as treatment targets and biomarkers, the mechanisms of exosomes in complex tumor-associated processes require further elucidation.

In conclusion, in the present study, miRNA expression profiles between TNBC and healthy controls were produced and analyzed by bioinformatics, revealing that certain miRNAs may have important roles in various biological and pathological processes of TNBC. These results may provide useful biological information for the early diagnosis of TNBC patients. In addition, certain exosomal miRNAs may serve as novel biomarkers, as they were determined to be of predictive value for the recurrence of breast cancer compared with non-recurrence. Yet, further studies are required to reveal the functional significance of abnormally expressed miRNAs in breast cancer, which is one of our next major research goals.

\section{Acknowledgements}

The author would like to thank their other colleagues of the Center for Precision Medicine, Meizhou People's Hospital who were not listed in the authorship for their helpful comments on the manuscript.

\section{Funding}

This study was supported by Medical and Health Research Project of Meizhou City, Guangdong Province, China (grant no. 2016-B-33 to QW) and the Science and Technology Planning Program of Meizhou City, Guangdong Province, China (grant no. 2015B037 to HZ).

\section{Availability of data and materials}

The data used to support the findings of this study are available from the corresponding author upon request.

\section{Authors' contributions}

HW and QW conceived and designed the experiments. HZ, LL, QZ, QH and ZY recruited subjects and collected and analyzed the clinical data. HW and ZY conducted the laboratory testing. ZY and HZ helped to analyze the data. HW and QW prepared the manuscript. HW reviewed the manuscript. All authors read and approved the manuscript and agree to be accountable for all aspects of the research in ensuring that the accuracy or integrity of any part of the work are appropriately investigated and resolved.

\section{Ethics approval and consent to participate}

This study was conducted on the basis of the Declaration of Helsinki, and was supported by the Ethics Committee of the Meizhou People's Hospital (Meizhou, Guangdong, China).

\section{Patient consent for publication}

Not applicable.

\section{Competing interests}

The authors declare that they have no competing interests.

\section{References}

1. Siegel RL, Ma J, Zou Z and Jemal A: Cancer statistics, 2014. CA Cancer J Clin 64: 9-29, 2014.

2. DeSantis C, Ma J, Bryan L and Jemal A: Breast cancer statistics, 2013. CA Cancer J Clin 64: 52-62, 2014. 
3. Unger-Saldaña $\mathrm{K}$ : Challenges to the early diagnosis and treatment of breast cancer in developing countries. World J Clin Onco 5: 465-477, 2014.

4. Fan L, Strasser-Weippl K, Li JJ, St Louis J, Finkelstein DM, Yu KD, Chen WQ, Shao ZM and Goss PE: Breast cancer in China. Lancet Oncol 15: e279-e289, 2014.

5. Wiechmann L, Sampson M, Stempel M, Jacks LM, Patil SM, King T and Morrow M: Presenting features of breast cancer differ by molecular subtype. Ann Surg Oncol 16: 2705-2710, 2009.

6. Parise CA, Bauer KR, Brown MM and Caggiano V: Breast cancer subtypes as defined by the estrogen receptor (ER), progesterone receptor (PR), and the human epidermal growth factor receptor 2 (HER2) among women with invasive breast cancer in California, 2004. Breast J 15: 593-602, 2009.

7. Hugh J, Hanson J, Cheang MC, Nielsen TO, Perou CM, Dumontet C, Reed J, Krajewska M, Treilleux I, Rupin M, et al Breast cancer subtypes and response to docetaxel in node-positive breast cancer: Use of an immunohistochemical definition in the BCIRG 001 trial. J Clin Oncol 27: 1168-1178, 2009.

8. Park S, Koo JS, Min SK, Park HS, Lee JS, Lee JS, Kim SI and Park BW: Characteristics and outcomes according to molecular subtypes of breast cancer as classified by a panel of four biomarkers using immunohistochemistry. Breast 21: 50-57, 2012.

9. Meyers MO, Klauber-Demore N, Ollila DW, Amos KD Moore DT, Drobish AA, Burrows EM, Dees EC and Carey LA: Impact of breast cancer molecular subtypes on locoregional recurrence in patients treated with neoadjuvant chemotherapy for locally advanced breast cancer. Ann Surg Oncol 18: 2851-2857, 2011.

10. Sørlie T, Perou CM, Tibshirani R, Aas T, Geisler S, Johnsen H, Hastie T, Eisen MB, van de Rijn M, Jeffrey SS, et al: Gene expression patterns of breast carcinomas distinguish tumor subclasses with clinical implications. Proc Natl Acad Sci USA 98: 10869-10874, 2001.

11. Sorlie T, Tibshirani R, Parker J, Hastie T, Marron JS, Nobel A, Deng S, Johnsen H, Pesich R, Geisler S, et al: Repeated observation of breast tumor subtypes in independent gene expression data sets. Proc Natl Acad Sci USA 100: 8418-8423, 2003.

12. Badve S, Turbin D, Thorat MA, Morimiya A, Nielsen TO, Perou CM, Dunn S, Huntsman DG and Nakshatri H: FOXA1 expression in breast cancer-correlation with luminal subtype A and survival. Clin Cancer Res 13: 4415-4421, 2007.

13. Tang LC, Jin X, Yang HY, He M, Chang H, Shao ZM and Di GH: Luminal B subtype: A key factor for the worse prognosis of young breast cancer patients in China. BMC Cancer 15 201, 2015.

14. Böcker W, Moll R, Poremba C, Holland R, Van Diest PJ, Dervan P, Bürger H, Wai D, Ina Diallo R, Brandt $\mathrm{B}$, et al: Common adult stem cells in the human breast give rise to glandular and myoepithelial cell lineages: A new cell biological concept. Lab Invest 82: 737-746, 2002.

15. Birnbaum D, Bertucci F, Ginestier C, Tagett R, Jacquemier J and Charafe-Jauffret E: Basal and luminal breast cancers: Basic or luminous. Int J Oncol 25: 249-258, 2004.

16. Turner NC, Reisfilho JS, Russell AM, Springall RJ, Ryder K, Steele D, Savage K, Gillett CE, Schmitt FC, Ashworth A and Tutt AN: BRCA1 dysfunction in sporadic basal-like breast cancer. Oncogene 26: 2126-2132, 2007.

17. Millikan RC, Newman B, Tse CK, Moorman PG, Conway K, Dressler LG, Smith LV, Labbok MH, Geradts J, Bensen JT, et al: Epidemiology of basal-like breast cancer. Breast Cancer Res Treat 109: 123-139, 2008.

18. Thike AA, Cheok PY, Jaralazaro AR, Tan B, Tan P and Tan PH Triple-negative breast cancer: Clinicopathological characteristics and relationship with basal-like breast cancer. Mod Pathol 23 . 123-133, 2010

19. Rakha E, Ellis I and Reis-Filho J: Are triple-negative and basal-like breast cancer synonymous? Clin Cancer Res 14 618-619, 2008

20. Chacón RD and Costanzo MV: Triple-negative breast cancer. Breast Cancer Res 12 (Suppl 2): S3, 2010.

21. Podo F, Buydens LM, Degani H, Hilhorst R, Klipp E, Gribbestad IS, Van Huffel S, van Laarhoven HW, Luts J, Monleon D, et al: Triple-negative breast cancer: Present challenges and new perspectives. Mol Oncol 4: 209-229, 2010.

22. Oakman C, Moretti E, Galardi F, Biagioni C, Santarpia L, Biganzoli L and Di Leo A: Adjuvant systemic treatment for individual patients with triple negative breast cancer. Breast 20 (Suppl 3): S135-S141, 2011.
23. André F and Zielinski CC: Optimal strategies for the treatment of metastatic triple-negative breast cancer with currently approved agents. Ann Oncol 23 (Suppl 6): vi46-vi51, 2012.

24. Chevillet JR, Kang Q, Ruf IK, Briggs HA, Vojtech LN, Hughes SM, Cheng HH, Arroyo JD, Meredith EK, Gallichotte EN, et al: Quantitative and stoichiometric analysis of the microRNA content of exosomes. Proc Natl Acad Sci USA 111: 14888, 2014.

25. Wolfers J, Lozier A, Raposo G, Regnault A, Théry C, Masurier C, Flament C, Pouzieux S, Faure F, Tursz T, et al: Tumor-derived exosomes are a source of shared tumor rejection antigens for CTL cross-priming. Nat Med 7: 297-303, 2001.

26. Clayton A, Mitchell JP, Court J, Mason MD and Tabi Z Human tumor-derived exosomes selectively impair lymphocyte responses to interleukin-2. Cancer Res 67: 7458-7466, 2007.

27. Clayton A, Mitchell JP, Court J, Linnane S, Mason MD and Tabi Z: Human tumor-derived exosomes down-modulate NKG2D expression. J Immunol 180: 7249, 2008.

28. Hannafon BN and Ding WQ: Intercellular communication by exosome-derived microRNAs in cancer. Int J Mol Sci 14: 14240-14269, 2013.

29. Chaput N, Taïeb J, Schartz NE, André F, Angevin E and Zitvogel L: Exosome-based immunotherapy. Cancer Immunol Immun 53: 234-239, 2004.

30. Chendrimada TP, Gregory RI, Kumaraswamy E, Norman J, Cooch N, Nishikura K and Shiekhattar R: TRBP recruits the Dicer complex to Ago2 for microRNA processing and gene silencing. Nature 436: 740-744, 2005.

31. Hagiwara K, Kosaka N, Yoshioka Y, Takahashi RU, Takeshita $\mathrm{F}$ and Ochiya T: Stilbene derivatives promote Ago2-dependent tumour-suppressive microRNA activity. Sci Rep 2: 314, 2012

32. Bartel DP: MicroRNAs: Genomics, biogenesis, mechanism, and function. Cell 116: 281-297, 2004.

33. Livak KJ and Schmittgen TD: Analysis of relative gene expression data using real-time quantitative PCR and the 2(-Delta Delta $\mathrm{C}(\mathrm{T}))$ method. Methods 25: 402-408, 2001.

34. Zhang J, Jiang C, Shi X, Yu H, Lin H and Peng Y: Diagnostic value of circulating miR-155, miR-21, and miR-10b as promising biomarkers in human breast cancer. Int J Clin Exp Med 9: $10258-10265,2016$.

35. Shan HC and Toyokuni S: Malignant mesothelioma as an oxidative stress-induced cancer: An update. Free Radical Bio Med 86: 166-178, 2015.

36. Wang W and Luo YP: MicroRNAs in breast cancer: Oncogene and tumor suppressors with clinical potential. J Zhejiang Univer B 16: 18-31, 2015

37. Li X, Xin S, He Z, Che X, Wang J, Xiao X, Chen J and Song X: MicroRNA-21 (miR-21) post-transcriptionally downregulates tumor suppressor PDCD4 and promotes cell transformation, proliferation, and metastasis in renal cell carcinoma. Cell Physiol Biochem 33: 1631-1642, 2014

38. Corcoran C, Friel AM, Duffy MJ, Crown J and O'Driscoll L: Intracellular and extracellular microRNAs in breast cancer. Clin Chem 57: 18-32, 2011

39. Yong L, Jing Z, Zhang PY, Zhang Y, Sun SY, Yu SY and Xi QS: MicroRNA-10b targets E-cadherin and modulates breast cancer metastasis. Med Sci Monit 18: BR299-BR308, 2012.

40. Guttilla IK and White BA: Coordinate regulation of FOXO1 by miR-27a, miR-96, and miR-182 in breast cancer cells. J Biol Chem 284: 23204-23216, 2009.

41. Chen B, Tang H, Liu X, Liu P, Yang L, Xie X, Ye F, Song C, Xie X and Wei W: miR-22 as a prognostic factor targets glucose transporter protein type 1 in breast cancer. Cancer Lett 356: 410-417, 2015.

42. Eades G, Yang M, Yao Y, Zhang Y and Zhou Q: miR-200a regulates Nrf2 activation by targeting Keapl mRNA in breast cancer cells. J Biol Chem 286: 40725-40733, 2011.

43. Liu M, Hu C, Xu Q, Chen L, Ma K, Xu N and Zhu H: Methylseleninic acid activates Keap1/Nrf2 pathway via up-regulating miR-200a in human oesophageal squamous cell carcinoma cells. Bioscience Rep 35: e00256, 2015.

44. Tao S, He H, Chen Q and Yue W: GPER mediated estradiol reduces miR-148a to promote HLA-G expression in breast cancer. Biochem Biophys Res Commun 451: 74-78, 2014.

45. Tao S, He H and Chen Q: Estradiol induces HOTAIR levels via GPER-mediated miR-148a inhibition in breast cancer. J Trans Med 13: 131, 2015.

46. Xu X, Zhang Y, Jasper J, Lykken E, Alexander PB, Markowitz GJ, McDonnell DP, Li QJ and Wang XF: miR-148a functions to suppress metastasis and serves as a prognostic indicator in triple-negative breast cancer. Oncotarget 7: 20381-20394, 2016. 
47. Müller V, Gade S, Steinbach B, Loibl S, von Minckwitz G, Untch M, Schwedler K, Lübbe K, Schem C, Fasching PA, et al: Changes in serum levels of miR-21, miR-210, and miR-373 in HER2-positive breast cancer patients undergoing neoadjuvant therapy: A translational research project within the Geparquinto trial. Breast Cancer Res Treat 147: 61-68, 2014.

48. Hong L, Yang J, Han Y, Lu Q, Cao J and Syed L: High expression of miR-210 predicts poor survival in patients with breast cancer: A meta-analysis. Gene 507: 135-138, 2012.

49. Yin JY, Deng ZQ, Liu FQ, Qian J, Lin J, Tang Q, Wen XM, Zhou JD, Zhang YY and Zhu XW: Association between mir-24 and mir-378 in formalin-fixed paraffin-embedded tissues of breast cancer. Int J Clin Exp Patho 7: 4261-4267, 2014.

50. Ikeda K, Horieinoue K, Ueno T, Suzuki T, Sato W, Shigekawa T, Osaki A, Saeki T, Berezikov E, Mano H and Inoue S: miR-378a-3p modulates tamoxifen sensitivity in breast cancer MCF-7 cells through targeting GOLT1A. Sci Rep 5: 13170, 2015.

51. Savi F, Forno I, Faversani A, Luciani A, Caldiera S, Gatti S, Foa P, Ricca D, Bulfamante G, Vaira V and Bosari S: miR-296/Scribble axis is deregulated in human breast cancer and miR-296 restoration reduces tumour growth in vivo. Clin Sci (Lond) 127: 233-242, 2014.

52. Yang H, Yu J, Wang L, Ding D, Zhang L, Chu C, Chen Q, Xu Z, Zou Q and Liu X: miR-320a is an independent prognostic biomarker for invasive breast cancer. Oncol Lett 8: 1043-1050, 2014.

53. Yu J, Wang JG, Zhang L, Yang HP, Wang L, Ding D, Chen Q, Yang WL, Ren KH, Zhou DM, et al: MicroRNA-320a inhibits breast cancer metastasis by targeting metadherin. Oncotarget 7: 38612-38625, 2016.

54. Li L, Yuan L, Luo J, Gao J, Guo J and Xie X: miR-34a inhibits proliferation and migration of breast cancer through down-regulation of Bcl-2 and SIRT1. Clin Exp Med 13: 109-117, 2013.

55. Chabre O, Libé R, Assie G, Barreau O, Bertherat J, Bertagna X, Feige JJ and Cherradi N: Serum miR-483-5p and miR-195 are predictive of recurrence risk in adrenocortical cancer patients. Endocr Relat Cancer 20: 579-594, 2013.

56. Rask L, Balslev E, Søkilde R, Høgdall E, Flyger H, Eriksen J and Litman T: Differential expression of miR-139, miR-486 and miR-21 in breast cancer patients sub-classified according to lymph node status. Cell Oncol 37: 215-227, 2014

57. Song Q, Xu Y, Yang C, Chen Z, Jia C, Chen J, Zhang Y, Lai P, Fan X, Zhou X, et al: miR-483-5p promotes invasion and metastasis of lung adenocarcinoma by targeting RhoGDI1 and ALCAM. Cancer Res 74: 3031-3042, 2014

58. Zhang M, Liu D, Li W, Wu X, Gao CE and Li X: Identification of featured biomarkers in breast cancer with microRNA microarray. Arch Gynecol Obstet 294: 1047-1053, 2016.

59. Jia Z, Liu Y, Gao Q, Han Y, Zhang G, Xu S, Cheng K and Zou W: miR-490-3p inhibits the growth and invasiveness in triple-negative breast cancer by repressing the expression of TNKS2. Gene 593: 41-47, 2016.

60. Uen YH, Wang JW, Wang CC, Jhang Y, Chung JY, Tseng T, Sheu M and Lee S: Mining of potential microRNAs with clinical correlation-regulation of syndecan-1 expression by miR-122-5p altered mobility of breast cancer cells and possible correlation with liver injury. Oncotarget 9: 28165-28175, 2018

61. Block I, Burton M, Sørensen KP, Andersen L, Larsen MJ, Bak M, Cold S, Thomassen M, Tan Q and Kruse TA: Association of miR-548c-5p, miR-7-5p, miR-210-3p, miR-128-3p with recurrence in systemically untreated breast cancer. Oncotarget 9 : 9030-9042, 2018.

62. Wang B, Yang Z, Wang H, Cao Z, Zhao Y, Gong C, Ma L, Wang X, Hu X and Chen S: MicroRNA-320a inhibits proliferation and invasion of breast cancer cells by targeting RAB11A Am J Cancer Res 5: 2719-2729, 2015.

63. Frères P, Bouznad N, Servais L, Josse C, Wenric S, Poncin A, Thiry J, Moonen M, Oury C, Lancellotti P, et al: Variations of circulating cardiac biomarkers during and after anthracycline-containing chemotherapy in breast cancer patients. BMC Cancer 18: 102, 2018.

64. Hamdi K, Goerlitz D, Stambouli N, Islam M, Baroudi O, Neili B Benayed F, Chivi S, Loffredo C, Jillson IA, et al: miRNAs in Sera of Tunisian patients discriminate between inflammatory breast cancer and non-inflammatory breast cancer. SpringerPlus 3: 636, 2014.

65. Liu Y, Cai Q, Bao PP, Su Y, Cai H, Wu J, Ye F, Guo X, Zheng W, Zheng Y and Shu XO: Tumor tissue microRNA expression in association with triple-negative breast cancer outcomes. Breast Cancer Res Treat 152: 183-191, 2015.

66. Fassan M, Baffa R, Palazzo JP, Lloyd J, Crosariol M, Liu CG, Volinia S, Alder H, Rugge M, Croce CM and Rosenberg A: MicroRNA expression profiling of male breast cancer. Breast Cancer Res 11: R58, 2009.
67. Farazi TA, Horlings HM, Ten Hoeve JJ, Mihailovic A, Halfwerk H, Morozov P, Brown M, Hafner M, Reyal F, van Kouwenhove M, et al: MicroRNA sequence and expression analysis in breast tumors by deep sequencing. Cancer Res 71: 4443-4453, 2011.

68. Ouyang M, Li Y, Ye S, Ma J, Lu L, Lv W, Chang G, Li X, Li Q, Wang S and Wang W: MicroRNA profiling implies new markers of chemoresistance of triple-negative breast cancer. PLoS One 9: e96228, 2014

69. Cao ZG, Huang YN, Yao L, Liu YR, Hu X, Hou YF and Shao ZM: Positive expression of miR-361-5p indicates better prognosis for breast cancer patients. J Thorac Dis 8: 1772-1779, 2016.

70. Han J, Yu J, Dai Y, Li J, Guo M, Song J and Zhou X: Overexpression of miR-361-5p in triple-negative breast cancer (TNBC) inhibits migration and invasion by targeting RQCD1 and inhibiting the EGFR/PI3K/Akt pathway. Bosn J Basic Med Sci 19: 52-59, 2019.

71. Chang YY, Kuo WH, Hung JH, Lee CY, Lee YH, Chang YC Lin WC, Shen CY, Huang CS, Hsieh FJ, et al: Deregulated microRNAs in triple-negative breast cancer revealed by deep sequencing. Mol Cancer 14: 36, 2015.

72. McFall T,Mcknight B,RosatiR,Kim S,Huang Y, Viola-Villegas N and Ratnam M: Progesterone receptor A promotes invasiveness and metastasis of luminal breast cancer by suppressing regulation of critical microRNAs by estrogen. J Biol Chem 293: 1163-1177, 2018.

73. Benvenuti S, Sartorebianchi A, Di Nicolantonio F, Zanon C, Moroni M, Veronese S, Siena S and Bardelli A: Oncogenic activation of the RAS/RAF signaling pathway impairs the response of metastatic colorectal cancers to anti-epidermal growth factor receptor antibody therapies. Cancer Res 67: 2643-2648, 2007.

74. Downward J: Targeting RAS signalling pathways in cancer therapy. Nat Rev Cancer 3: 11-22, 2003.

75. Guertin DA and Sabatini DM: Defining the role of mTOR in cancer. Cancer Cell 12: 9-22, 2007.

76. Zoncu R, Efeyan A and Sabatini DM: mTOR: From growth signal integration to cancer, diabetes and ageing. Nat Rev Mol Cell Biol 12: 21-35, 2011.

77. Biankin AV, Waddell N, Kassahn KS, Gingras MC, Muthuswamy LB, Johns AL, Miller DK, Wilson PJ, Patch AM, Wu J, et al: Pancreatic cancer genomes reveal aberrations in axon guidance pathway genes. Nature 491: 399-405, 2012.

78. Kidd PM: The use of mushroom glucans and proteoglycans in cancer treatment. Altern Med Rev 5: 4-27, 2000.

79. Iozzo RV and Sanderson RD: Proteoglycans in cancer biology, tumour microenvironment and angiogenesis. J Cell Mol Med 15: 1013-1031, 2011.

80. Van Brocklyn JR: Sphingolipid signaling pathways as potential therapeutic targets in gliomas. Mini Rev Med Chem 7: 984-990, 2007.

81. Yuan HD, Quan HY, Zhang Y, Kim SH and Chung SH: 20(S)-Ginsenoside Rg3-induced apoptosis in HT-29 colon cancer cells is associated with AMPK signaling pathway. Mol Med Rep 3: 825-831, 2010.

82. Green AS, Chapuis N, Lacombe C, Mayeux P, Bouscary D and Tamburini J: LKB1/AMPK/mTOR signaling pathway in hematological malignancies: From metabolism to cancer cell biology. Cell Cycle 10: 2115-2120, 2011

83. Dentice M, Luongo C, Ambrosio R, Sibilio A, Casillo A, Iaccarino A, Troncone G, Fenzi G, Larsen PR and Salvatore D: $\beta$-catenin regulates deiodinase levels and thyroid hormone signaling in colon cancer cells. Gastroenterol 143: 1037-1047, 2012.

84. King CR, Kraus MH and Aaronson SA: Amplification of a novel $\mathrm{v}$-erbB-related gene in a human mammary carcinoma. Science 229: 974-976, 1985 .

85. Normanno N, De Luca A, Maiello MR, Campiglio M, Napolitano M, Mancino M, Carotenuto A, Viglietto $G$ and Menard S: The MEK/MAPK pathway is involved in the resistance of breast cancer cells to the EGFR tyrosine kinase inhibitor gefitinib. J Cell Physiol 207: 420-427, 2006.

86. Oxnard GR, Arcila ME, Sima CS, Riely GJ, Chmielecki J, Kris MG, Pao W, Ladanyi M and Miller V: Acquired resistance to EGFR tyrosine kinase inhibitors in EGFR mutant lung cancer: Distinct natural history of patients with tumors harboring the T790M mutation. Clin Cancer Res 17: 1616-1622, 2011.

This work is licensed under a Creative Commons Attribution-NonCommercial-NoDerivatives 4.0 International (CC BY-NC-ND 4.0) License. 